\title{
Boundedness for multilinear commutator of Marcinkiewicz operator with variable kernels on Hardy and Herz-Hardy spaces
}

\author{
Wenxin $\mathrm{Yu}^{1 *}$, Yigang He $\mathrm{He}^{1,2}$, Qiwu Luo ${ }^{1}$, Jian Zheng ${ }^{1}$ and Xianming $\mathrm{Wu}^{1}$
}

\section{"Correspondence:}

slowbird@sohu.com

${ }^{1}$ College of Electrical and

Information Engineering, Hunan

University, Changsha, Hunan

410082, P.R. China

Full list of author information is

available at the end of the article

\begin{abstract}
In this paper, the $\left(H_{\vec{b}^{\prime}}^{p} L^{p}\right)$ - and $\left(H \dot{K}_{q, \vec{b}}^{\alpha, p}, \dot{K}_{q}^{\alpha, p}\right)$-type boundedness for the multilinear commutator related to the Marcinkiewicz operator with variable kernels is obtained.
\end{abstract} MSC: 42B20; 42B25

Keywords: Marcinkiewicz operator; multilinear commutator; BMO; Hardy space; Herz-Hardy space

\section{Introduction and definitions}

Let $T$ be the Calderón-Zygmund operator and $b \in B M O\left(R^{n}\right)$. The commutator $[b, T]$ generated by $T$ and $b$ is defined by

$$
[b, T](f)(x)=b(x) T(f)(x)-T(b f)(x) .
$$

A classical result of Coifman, Rochberg and Weiss (see $[1,2])$ proved that the commutator $[b, T]$ is bounded on $L^{p}\left(R^{n}\right)(1<p<\infty)$. However, it was observed that the $[b, T]$ is not bounded, in general, from $H^{p}\left(R^{n}\right)$ to $L^{p}\left(R^{n}\right)$. But if $H^{p}\left(R^{n}\right)$ is replaced by a suitable atomic space $H_{b}^{P}\left(R^{n}\right)$, then $[b, T]$ maps continuously $H_{b}^{P}\left(R^{n}\right)$ into $L^{p}\left(R^{n}\right)$ (see [3]). In addition, we easily know that $H_{b}^{p}\left(R^{n}\right) \subset H^{p}\left(R^{n}\right)$. In recent years, the theory of Herz-type Hardy spaces have been developed (see [4-7]). The main purpose of this paper is to consider the continuity of multilinear commutators related to Marcinkiewicz operators with variable kernels and $B M O\left(R^{n}\right)$ functions on certain Hardy and Herz-Hardy spaces. Let us first introduce some definitions (see [3-14]).

Given a positive integer $m$ and $1 \leq j \leq m$, we denote by $C_{j}^{m}$ the family of all finite subsets $\sigma=\{\sigma(1), \ldots, \sigma(j)\}$ of $\{1, \ldots, m\}$ of $j$ different elements. For $\sigma \in C_{j}^{m}$, set $\sigma^{c}=\{1, \ldots, m\} \backslash \sigma$. For $\vec{b}=\left(b_{1}, \ldots, b_{m}\right)$ and $\sigma=\{\sigma(1), \ldots, \sigma(j)\} \in C_{j}^{m}$, set $\vec{b}_{\sigma}=\left(b_{\sigma(1)}, \ldots, b_{\sigma(j)}\right), b_{\sigma}=b_{\sigma(1)} \cdots b_{\sigma(j)}$ and $\left\|\vec{b}_{\sigma}\right\|_{B M O}=\left\|b_{\sigma(1)}\right\|_{B M O} \cdots\left\|b_{\sigma(j)}\right\|_{B M O}$.

Definition 1 Let $b_{i}(i=1, \ldots, m)$ be a locally integrable function and $0<p \leq 1$. A bounded measurable function $a$ on $R^{n}$ is said to be a $(p, \vec{b})$ atom if

(1) $\operatorname{supp} a \subset B=B\left(x_{0}, r\right)$,

(2) $\|a\|_{L^{\infty}} \leq|B|^{-1 / p}$,

(3) $\int_{B} a(y) d y=\int_{B} a(y) \prod_{l \in \sigma} b_{l}(y) d y=0$ for any $\sigma \in C_{j}^{m}, 1 \leq j \leq m$.

( 2012 Yu et al.; licensee Springer. This is an Open Access article distributed under the terms of the Creative Commons Attribution License (http://creativecommons.org/licenses/by/2.0), which permits unrestricted use, distribution, and reproduction in any medium, provided the original work is properly cited. 
A temperate distribution $f$ is said to belong to $H_{\vec{b}}^{p}\left(R^{n}\right)$ if, in the Schwartz distribution sense, it can be written as

$$
f(x)=\sum_{j=1}^{\infty} \lambda_{j} a_{j}(x)
$$

where every $a_{j}$ is $(p, \vec{b})$ atom, $\lambda \in C$ and $\sum_{j=1}^{\infty}|\lambda|^{p}<\infty$. Moreover, $\|f\|_{H_{\vec{b}}^{p}\left(R^{n}\right)} \approx$ $\left(\sum_{j=1}^{\infty}\left|\lambda_{j}\right|^{p}\right)^{1 / p}$

Given a set $E \subset R^{n}$, the characteristic function of $E$ is defined by $\chi_{E}$. Let $B_{k}=\left\{x \in R^{n}\right.$ : $\left.|x| \leq 2^{k}\right\}$ and $C_{k}=B_{k} \backslash B_{k-1}$ and $\chi_{k}=\chi_{B_{k}}, k \in Z$.

Definition 2 Let $0<p, q<\infty, \alpha \in R$. For $k \in Z$, set $B_{k}=\left\{x \in R^{n}:|x| \leq 2^{k}\right\}$ and $C_{k}=B_{k} \backslash$ $B_{k-1}$. Denote by $\chi_{k}$ the characteristic function of $C_{k}$ and by $\chi_{0}$ the characteristic function of $B_{0}$.

(1) The homogeneous Herz space is defined by

$$
\dot{K}_{q}^{\alpha, p}\left(R^{n}\right)=\left\{f \in L_{\mathrm{loc}}^{q}\left(R^{n} \backslash\{0\}\right):\|f\|_{\left.\dot{K}_{q}^{\alpha, p}<\infty\right\}}\right.
$$

where

$$
\|f\|_{\dot{K}_{q}^{\alpha, p}}=\left[\sum_{k=-\infty}^{\infty} 2^{k \alpha p}\left\|f \chi_{k}\right\|_{L^{q}}^{p}\right]^{1 / p} .
$$

(2) The nonhomogeneous Herz space is defined by

$$
K_{q}^{\alpha, p}\left(R^{n}\right)=\left\{f \in L_{\mathrm{loc}}^{q}\left(R^{n}\right):\|f\|_{\left.K_{q}^{\alpha, p}<\infty\right\}},\right.
$$

where

$$
\|f\|_{K_{q}^{\alpha, p}}=\left[\sum_{k=1}^{\infty} 2^{k \alpha p}\left\|f \chi_{k}\right\|_{L^{q}}^{p}+\left\|f \chi_{0}\right\|_{L^{q}}^{p}\right]^{1 / p} .
$$

Definition 3 Let $\alpha \in R, 1<q<\infty, 0<\alpha<n(1-1 / q), b_{i} \in B M O\left(R^{n}\right), 1 \leq i \leq m$. A function $a$ on $R^{n}$ is called a central $(\alpha, q, \vec{b})$-atom (or a central $(\alpha, q, \vec{b})$-atom of restrict type) if

(1) $\operatorname{supp} a \subset B=B(0, r)$ (or for some $r \geq 1$ ),

(2) $\|a\|_{L^{q}} \leq|B|^{-\alpha / n}$,

(3) $\int_{B} a(x) d x=\int_{B} a(x) \prod_{l \in \sigma} b_{l}(x) d x=0$ for any $\sigma \in C_{j}^{m}, 1 \leq j \leq m$.

A temperate distribution $f$ is said to belong to $H \dot{K}_{q, \vec{b}}^{\alpha, p}\left(R^{n}\right)$ (or $H K_{q, \vec{b}}^{\alpha, p}\left(R^{n}\right)$ ) if it can be written as $f=\sum_{j=-\infty}^{\infty} \lambda_{j} a_{j}$ (or $f=\sum_{j=0}^{\infty} \lambda_{j} a_{j}$ ), in the Schwartz distribution sense, where $a_{j}$ is a central $(\alpha, q, \vec{b})$-atom (or a central $(\alpha, q, \vec{b})$-atom of restrict type) supported on $B\left(0,2^{j}\right)$ and $\sum_{-\infty}^{\infty}\left|\lambda_{j}\right|^{p}<\infty$ (or $\left.\sum_{j=0}^{\infty}\left|\lambda_{j}\right|^{p}<\infty\right)$. Moreover, $\|f\|_{H \dot{K}_{q, \vec{b}}^{\alpha, p}}\left(\right.$ or $\left.\|f\|_{H K_{q, \vec{b}}^{\alpha, p}}\right) \approx\left(\sum_{j}\left|\lambda_{j}\right|^{p}\right)^{1 / p}$.

Definition 4 Let $\Omega$ be homogeneous of degree zero on $R^{n}$ such that $\omega_{r}(\delta)$ is defined

$$
\omega_{r}(\delta)=\sup _{|\rho|<\delta}\left(\int_{S^{n-1}}|\Omega(\rho \hat{x})-\Omega(\dot{x})|^{r} d \sigma(\dot{x})\right)^{1 / r},
$$

where $|\rho|=\sup _{\dot{x} \in S^{n-1}}|\rho \dot{x}-x|$. 
If $\int_{0}^{1} \frac{\omega_{r}(\delta)}{\delta} d \delta<\infty$, we say $\Omega(x)$ satisfied $L^{r}$-Dini condition.

Definition 5 Let $0<\epsilon<n, 0<\gamma \leq 1$ and $\Omega$ be homogeneous of degree zero on $R^{n}$ such that $\int_{S^{n-1}} \Omega\left(x^{\prime}\right) d \sigma\left(x^{\prime}\right)=0$. Assume that $\Omega \in \operatorname{Lip}_{\gamma}\left(S^{n-1}\right)$, that is, there exists a constant $M>$ 0 such that for any $x, y \in S^{n-1},|\Omega(x)-\Omega(y)| \leq M|x-y|^{\gamma}$. The Marcinkiewicz multilinear commutator is defined by

$$
\mu_{\epsilon}^{\tilde{b}}(f)(x)=\left(\int_{0}^{\infty}\left|F_{t}^{\tilde{b}}(f)(x)\right|^{2} \frac{d t}{t^{3}}\right)^{1 / 2}
$$

where

$$
F_{t}^{\tilde{b}}(f)(x)=\int_{|x-y| \leq t} \frac{\Omega(x-y)}{|x-y|^{n-1-\epsilon}}\left[\prod_{j=1}^{m}\left(b_{j}(x)-b_{j}(y)\right)\right] f(y) d y .
$$

Set

$$
F_{t}(f)(x)=\int_{|x-y| \leq t} \frac{\Omega(x-y)}{|x-y|^{n-1-\epsilon}} f(y) d y
$$

we also define that

$$
\mu_{\epsilon}(f)(x)=\left(\int_{0}^{\infty}\left|F_{t}(f)(x)\right|^{2} \frac{d t}{t^{3}}\right)^{1 / 2}
$$

which is the Marcinkiewicz operator (see $[9,13,14])$.

\section{Theorems and proofs}

We begin with three preliminary lemmas.

Lemma 1 (see [12]) Let $1<r<\infty, b_{j} \in B M O\left(R^{n}\right)$ for $j=1, \ldots, k$ and $k \in N$. Then we have

$$
\frac{1}{|Q|} \int_{Q} \prod_{j=1}^{k}\left|b_{j}(y)-\left(b_{j}\right)_{Q}\right| d y \leq C \prod_{j=1}^{k}\left\|b_{j}\right\|_{B M O\left(R^{n}\right)}
$$

and

$$
\left(\frac{1}{|Q|} \int_{Q} \prod_{j=1}^{k}\left|b_{j}(y)-\left(b_{j}\right)_{Q}\right|^{r} d y\right)^{1 / r} \leq C \prod_{j=1}^{k}\left\|b_{j}\right\|_{B M O\left(R^{n}\right)} .
$$

Lemma 2 (see [14]) Let $0<\epsilon<n, 1<s<n / \epsilon$ and $1 / r=1 / s-\epsilon / n$. Then $\mu_{\epsilon}^{\vec{b}}$ is bounded from $L^{s}\left(R^{n}\right)$ to $L^{r}\left(R^{n}\right)$.

Lemma 3 (see [15]) Let $0<\mu<n, \Omega(x, z) \in L^{\infty}\left(R^{n}\right)$ satisfy $L^{r}\left(S^{n-1}\right)(r \geq 1)$ conditions, that is, there exists a constant $0<a_{0}<1 / 2$ such that for $\left|y_{0}\right|<a_{0} R$,

$$
\left(\int_{R<|x|<2 R}\left|\frac{\Omega(x, x-y)}{|x-y|^{n-\mu}}-\frac{\Omega(x, x)}{|x-y|^{n-\mu}}\right|^{r} d x\right)^{1 / r} \leq C R^{n / r-(n-\mu)}\left(\frac{|y|}{R}+\int_{|y| / 2 R}^{|y| / R} \frac{\omega_{r}(\delta)}{\delta} d \delta\right) .
$$


Theorem 1 Let $0<\epsilon<n, n /(n+1 / 2-\epsilon)<q \leq 1,1 / q=1 / p-\epsilon / n, \vec{b}=\left(b_{1}, \ldots, b_{m}\right), b_{i} \in B M O$, $1 \leq i \leq m$. Then $\mu_{\epsilon}^{\vec{b}}$ is bounded from $H_{\vec{b}}^{p}\left(R^{n}\right)$ to $L^{q}\left(R^{n}\right)$.

Proof It suffices to show that there exists a constant $C>0$ such that for every $(p, \vec{b})$ atom $a$,

$$
\left\|\mu_{\epsilon}^{\vec{b}}(a)\right\|_{L^{q}} \leq C
$$

Let $a$ be a $(p, \vec{b})$ atom supported on a ball $B=B\left(x_{0}, 2 d\right)$. We write

$$
\int_{R^{n}}\left|\mu_{\epsilon}^{\vec{b}}(a)(x)\right|^{q} d x=\int_{\left|x-x_{0}\right| \leq 2 d}\left|\mu_{\epsilon}^{\vec{b}}(a)(x)\right|^{q} d x+\int_{\left|x-x_{0}\right|>2 d}\left|\mu_{\epsilon}^{\vec{b}}(a)(x)\right|^{q} d x=I+I I .
$$

For $I$, taking $r, s>1$ with $q<s<n / \epsilon$ and $1 / r=1 / s-\epsilon / n$, by Hölder's inequality and the $\left(L^{s}, L^{r}\right)$-boundedness of $\mu_{\epsilon}^{\vec{b}}$, we get

$$
I \leq C\left\|\mu_{\epsilon}^{\vec{b}}(a)\right\|_{L^{r}}^{q}\left|B\left(x_{0}, 2 d\right)\right|^{1-q / r} \leq C\|a\|_{L^{s}}^{q}|B|^{1-q / r} \leq C|B|^{-q / p+q / s+1-q / r} \leq C .
$$

For $I I$, denoting $\lambda=\left(\lambda_{1}, \ldots, \lambda_{m}\right)$ with $\lambda_{i}=\left(b_{i}\right)_{B}, 1 \leq i \leq m$, where $\left(b_{i}\right)_{B}=\left|B\left(x_{0}, 2 d\right)\right|^{-1} \times$ $\int_{B\left(x_{0}, 2 d\right)} b_{i}(x) d x$, by Hölder's inequality and the vanishing moment of $a$, we get

$$
\begin{aligned}
I I \leq & {\left[\int_{0}^{\left|x-x_{0}\right|+2 d}\left|\int_{|x-y|<t} \prod_{j=1}^{m}\left(b_{j}(x)-b_{j}(y)\right) a(y) \frac{\Omega(x, x-y)}{|x-y|^{n-1-\epsilon}} d y\right|^{2} \frac{d t}{t^{3}}\right]^{1 / 2} } \\
& +\left[\int_{\left|x-x_{0}\right|+2 d}^{\infty}\left|\int_{|x-y|<t} \prod_{j=1}^{m}\left(b_{j}(x)-b_{j}(y)\right) a(y) \frac{\Omega(x, x-y)}{|x-y|^{n-1-\epsilon}} d y\right|^{2} \frac{d t}{t^{3}}\right]^{1 / 2} \\
= & I I_{1}+I I_{2} .
\end{aligned}
$$

Note that $|x-y| \sim\left|x-x_{0}\right| \sim\left|x-x_{0}\right|+2 d$ for $\left|x-x_{0}\right|>2 d, y \in B$. For $1 / t+1 / r=1$, we have

$$
\begin{aligned}
I I_{1} \leq & C \int_{R^{n}}\left(\int_{|x-y|}^{\left|x-x_{0}\right|+2 d} \frac{d t}{t^{3}}\right)^{1 / 2} \prod_{j=1}^{m}\left|b_{j}(x)-b_{j}(y)\right||a(y)| \frac{|\Omega(x, x-y)|}{|x-y|^{n-1-\epsilon}} d y \\
\leq & C \int_{R^{n}}\left|\frac{1}{|x-y|^{2}}-\frac{1}{\left(\left|x-x_{0}\right|+2 d\right)^{2}}\right|^{1 / 2} \prod_{j=1}^{m}\left|b_{j}(x)-b_{j}(y)\right||a(y)| \frac{|\Omega(x, x-y)|}{|x-y|^{n-1-\epsilon}} d y \\
\leq & C \int_{R^{n}} \prod_{j=1}^{m}\left|b_{j}(x)-b_{j}(y)\right||a(y)| \frac{|\Omega(x, x-y)|}{|x-y|^{n-1-\varepsilon}} \frac{\left|y-x_{0}\right|^{1 / 2}}{\left|x-x_{0}\right|^{3 / 2}} d y \\
\leq & C \int_{R^{n}} \prod_{j=1}^{m}\left|b_{j}(x)-b_{j}(y)\right||a(y)| \frac{|\Omega(x, x-y)|}{\left|x-x_{0}\right|^{n+1 / 2-\epsilon}}\left|y-x_{0}\right|^{1 / 2} d y \\
\leq & C \sum_{j=1}^{m-1} \sum_{\sigma \in C_{j}^{m}} \frac{1}{\left|x-x_{0}\right|^{n+1 / 2-\epsilon}}\left(\int_{B}\left|(\vec{b}(y)-\lambda)_{\sigma^{c}}\right||a(y)||\Omega(x, x-y)|\left|y-x_{0}\right|^{1 / 2} d y\right) \\
& \times\left|(\vec{b}(x)-\lambda)_{\sigma}\right| \\
\leq & C \sum_{j=1}^{m-1} \sum_{\sigma \in C_{j}^{m}} \frac{1}{\left|x-x_{0}\right|^{n+1 / 2-\epsilon}}\left(\int_{B}\left(\left|(\vec{b}(y)-\lambda)_{\sigma^{c}}\right||a(y)|\left|y-x_{0}\right|^{1 / 2}\right)^{t} d y\right)^{1 / t}
\end{aligned}
$$




$$
\begin{aligned}
& \times\left(\int_{B}(|\Omega(x, x-y)|)^{r} d y\right)^{1 / r}\left|(\vec{b}(x)-\lambda)_{\sigma}\right| \\
\leq & C \sum_{j=1}^{m-1} \sum_{\sigma \in C_{j}^{m}} \frac{d^{n(1-1 / p+1 / t+1 / r+1 / 2 n)}}{\left|x-x_{0}\right|^{n+1 / 2-\epsilon}}\left\|\vec{b}_{\sigma^{c}}\right\|_{B M O} \cdot\|\Omega\|_{L^{\infty} \times L^{r}}\left|(\vec{b}(x)-\lambda)_{\sigma}\right| \\
\leq & C \sum_{j=1}^{m-1} \sum_{\sigma \in C_{j}^{m}} \frac{d^{n(-1 / p+1 / t+1 / r+1 / 2 n)}}{\left|x-x_{0}\right|^{n+1 / 2-\epsilon}}\left\|\vec{b}_{\sigma}\right\|_{B M O}\left|(\vec{b}(x)-\lambda)_{\sigma}\right|,
\end{aligned}
$$

so we have

$$
\begin{aligned}
& \sum_{k=1}^{\infty} \int_{2^{k+1}} d \geq\left|x-x_{0}\right|>2^{k} d\left|\sum_{j=1}^{m-1} \sum_{\sigma \in C_{j}^{m}} \frac{d^{n(-1 / p+1 / t+1 / r+1 / 2 n)}}{\left|x-x_{0}\right|^{n+1 / 2-\epsilon}}\left\|\vec{b}_{\sigma^{c}}\right\|_{B M O}(\vec{b}(x)-\lambda)_{\sigma}\right|^{q} d x \\
& \leq C \sum_{j=1}^{m-1} \sum_{\sigma \in C_{j}^{m}}\left\|\vec{b}_{\sigma c}\right\|_{B M O}^{q} \sum_{k=1}^{\infty} \int_{C_{k}}\left(\frac{d^{n(1-1 / p+1 / 2 n)}}{|x-y|^{n+1 / 2-\epsilon}}\right)^{q}\left|(\vec{b}(x)-\lambda)_{\sigma}\right|^{q} d x \\
& \leq C \sum_{j=1}^{m-1} \sum_{\sigma \in C_{j}^{m}}\left\|\vec{b}_{\sigma^{c}}\right\|_{B M O}^{q} \sum_{k=1}^{\infty}\left(\frac{d^{q n(1+1 / 2 n-1 / q-\delta / n)}}{\left|2^{k} d\right|^{q(n+1 / 2-\epsilon)}}\right)\left(2^{k} d\right)^{n} \\
& \quad \times\left(\frac{1}{\left|2^{k} B\right|} \int_{2^{k} B}\left|(\vec{b}(x)-\lambda)_{\sigma}\right| d x\right)^{q} \\
& \leq C \sum_{k=1}^{\infty} k^{q} 2^{-k(q(n+1 / 2-\epsilon)-n)}\|\vec{b}\|_{B M O}^{q} \\
& \leq C\|\vec{b}\|_{B M O}^{q} .
\end{aligned}
$$

For $I_{2}$, we can obtain

$$
\begin{aligned}
I I_{2} & =\int_{R^{n}}\left(\int_{\left|x-x_{0}\right|+2 d}^{\infty} \frac{d t}{t^{3}}\right)^{1 / 2}\left|\prod_{j=1}^{m}\left(b_{j}(x)-b_{j}(y)\right) a(y) \frac{\Omega(x, x-y)}{|x-y|^{n-1-\epsilon}} d y\right| \\
& \leq C\left|\int_{R^{n}} \prod_{j=1}^{m}\left(b_{j}(x)-b_{j}(y)\right) a(y)\right| \frac{\Omega(x, x-y)}{|x-y|^{n-1-\epsilon}}-\frac{\Omega(x, x)}{|x|^{n-1-\epsilon}}|d y| \frac{1}{\left|x-x_{0}\right|+2 d} \\
& \leq C \int_{R^{n}} \prod_{j=1}^{m}\left|b_{j}(x)-b_{j}(y)\right||a(y)|\left|\frac{\Omega(x, x-y)}{|x-y|^{n-\epsilon}}-\frac{\Omega(x, x)}{|x|^{n-\epsilon}}\right| d y \\
& \leq C \sum_{j=1}^{m-1} \sum_{\sigma \in C_{j}^{m}}\left|(\vec{b}(x)-\lambda)_{\sigma}\right| \int_{B}\left|(\vec{b}(y)-\lambda)_{\sigma^{c}}\right||a(y)|\left|\frac{\Omega(x, x-y)}{|x-y|^{n-\epsilon}}-\frac{\Omega(x, x)}{|x|^{n-\epsilon}}\right| d y .
\end{aligned}
$$

Thus, by Lemma 3, we have

$$
\begin{aligned}
& \left\{\sum_{k=1}^{\infty} \int_{2^{k+1} d \geq\left|x-x_{0}\right|>2^{k} d}\left|I I_{2}\right|^{q} d x\right\}^{1 / q} \\
& \leq C\left\{\sum_{j=1}^{m-1} \sum_{\sigma \in C_{j}^{m}} \sum_{k=1}^{\infty} \int_{C_{k}}\left|(\vec{b}(x)-\lambda)_{\sigma}\right|^{q}\right.
\end{aligned}
$$




$$
\begin{aligned}
& \left.\times\left|\int_{B}\right|(\vec{b}(y)-\lambda)_{\sigma^{c}}|| a(y)|| \frac{\Omega(x, x-y)}{|x-y|^{n-\epsilon}}-\frac{\Omega(x, x)}{|x|^{n-\epsilon}}|d y|^{q} d x\right\}^{1 / q} \\
& \leq C \sum_{j=1}^{m-1} \sum_{\sigma \in C_{j}^{m}} \sum_{k=1}^{\infty} \int_{B}\left|(\vec{b}(y)-\lambda)_{\sigma^{c}}\right||a(y)| \\
& \times\left(\int_{C_{k}}\left|(\vec{b}(x)-\lambda)_{\sigma}\right|^{q}\left|\frac{\Omega(x, x-y)}{|x-y|^{n-\epsilon}}-\frac{\Omega(x, x)}{|x|^{n-\epsilon}}\right|^{q} d x\right)^{1 / q} d y \\
& \leq C \sum_{j=1}^{m-1} \sum_{\sigma \in C_{j}^{m}} \sum_{k=1}^{\infty}\left|2^{k+1} B\right|^{1 / q-1 / r}\left\|\vec{b}_{\sigma}\right\|_{B M O} \\
& \times \int_{B}\left|(\vec{b}(y)-\lambda)_{\sigma^{c}}\right||a(y)|\left(\int_{C_{k}}\left|\frac{\Omega(x, x-y)}{|x-y|^{n-\epsilon}}-\frac{\Omega(x, x)}{|x|^{n-\epsilon}}\right|^{r} d x\right)^{1 / r} d y \\
& \leq C \sum_{j=1}^{m-1} \sum_{\sigma \in C_{j}^{m}} \sum_{k=1}^{\infty}\left|2^{k+1} d\right|^{n(1 / q-1 / r)}\left|2^{k} d\right|^{n / r-(n-\epsilon)}\left\|\vec{b}_{\sigma}\right\|_{B M O} \int_{B}\left|(\vec{b}(y)-\lambda)_{\sigma^{c}}\right||a(y)| \\
& \times\left\{\frac{|y|}{\left|2^{k} d\right|}+\int_{|y| /\left|2^{k+1} d\right|<\delta<|y| /\left|2^{k} d\right|} \frac{\omega_{r}(\delta)}{\delta} d \delta\right\} d y \\
& \leq C \sum_{j=1}^{m-1} \sum_{\sigma \in C_{j}^{m}} \sum_{k=1}^{\infty}\left|2^{k} d\right|^{n / q-n / r}\left|2^{k} d\right|^{n / r-(n-\epsilon+1 / 2)}\left\|\vec{b}_{\sigma}\right\|_{B M O} \\
& \times \int_{B}\left|(\vec{b}(y)-\lambda)_{\sigma^{c}}\right||a(y)| d y \\
& \leq C \sum_{k=1}^{\infty}|B|\left|2^{k} d\right|^{n / q-(n-\epsilon+1 / 2)}\left\|\vec{b}_{\sigma}\right\|_{B M O}\left\|\vec{b}_{\sigma^{c}}\right\|_{B M O} \\
& \leq C\|\vec{b}\|_{B M O}
\end{aligned}
$$

This finishes the proof of Theorem 1.

Theorem 2 Let $0<\epsilon<n, 0<p<\infty, 1<q_{1}, q_{2}<\infty, 1 / q_{1}-1 / q_{2}=\epsilon / n, n\left(1-1 / q_{1}\right) \leq \alpha<$ $n\left(1-1 / q_{1}\right)+1 / 2+\epsilon$ and $b_{i} \in B M O\left(R^{n}\right), 1 \leq i \leq m, \vec{b}=\left(b_{1}, \ldots, b_{m}\right)$. Then $\mu_{\epsilon}^{\vec{b}}$ is bounded from $H \dot{K}_{q_{1}, \vec{b}}^{\alpha, p}\left(R^{n}\right)$ to $\dot{K}_{q_{2}}^{\alpha, p}\left(R^{n}\right)$.

Proof Let $f \in H \dot{K}_{q, \vec{b}}^{\alpha, p}\left(R^{n}\right)$ and $f(x)=\sum_{j=-\infty}^{\infty} \lambda_{j} a_{j}(x)$ be the atomic decomposition for $f$ as in Definition 3. We write

$$
\begin{aligned}
& \left\|\mu_{\epsilon}^{\vec{b}}(f)(x)\right\|_{\dot{K}_{q_{2}}^{\alpha, p}}^{p} \\
& \leq \sum_{k=-\infty}^{\infty} 2^{k \alpha p}\left(\sum_{j=-\infty}^{k-3}\left|\lambda_{j}\right|\left\|\mu_{\epsilon}^{\vec{b}}\left(a_{j}\right)(x) \chi_{k}\right\|_{L^{q_{2}}}\right)^{p} \\
& \quad+\sum_{k=-\infty}^{\infty} 2^{k \alpha p}\left(\sum_{j=k-2}^{\infty}\left|\lambda_{j}\right|\left\|\mu_{\epsilon}^{\vec{b}}\left(a_{j}\right)(x) \chi_{k}\right\|_{L^{q_{2}}}\right)^{p} \\
& =J+J J .
\end{aligned}
$$


For $J J$, by the $\left(L^{q_{1}}, L^{q_{2}}\right)$-boundedness of $\mu_{\epsilon}^{\vec{b}}$, we get

$$
\begin{aligned}
& J \leq C \sum_{k=-\infty}^{\infty} 2^{k \alpha p}\left(\sum_{j=k-2}^{\infty}\left|\lambda_{j}\right|\left\|a_{j}\right\|_{L^{q_{1}}}\right)^{p} \\
& \leq C \sum_{k=-\infty}^{\infty} 2^{k \alpha p}\left(\sum_{j=k-2}^{\infty}\left|\lambda_{j}\right| 2^{-j \alpha}\right)^{p} \\
& \leq \begin{cases}C \sum_{k=-\infty}^{\infty} 2^{k \alpha p}\left(\sum_{j=k-2}^{\infty}\left|\lambda_{j}\right|^{p} 2^{-j \alpha p}\right), & 0<p \leq 1, \\
C \sum_{k=-\infty}^{\infty} 2^{k \alpha p}\left(\sum_{j=k-2}^{\infty}\left|\lambda_{j}\right|^{p} 2^{-j \alpha p / 2}\right)\left(\sum_{j=k-2}^{\infty} 2^{-j \alpha p^{\prime} / 2}\right)^{p / p^{\prime}}, & p>1,\end{cases} \\
& \leq \begin{cases}C \sum_{j=-\infty}^{\infty}\left|\lambda_{j}\right|^{p}\left(\sum_{k=-\infty}^{j+2} 2^{(k-j) \alpha p}\right), & 0<p \leq 1, \\
C \sum_{j=-\infty}^{\infty}\left|\lambda_{j}\right|^{p}\left(\sum_{k=-\infty}^{j+2} 2^{(k-j) \alpha p / 2}\right)\left(\sum_{k=-\infty}^{j+2} 2^{(k-j) \alpha p^{\prime} / 2}\right)^{p / p^{\prime}}, & p>1,\end{cases} \\
& \leq C \sum_{j=-\infty}^{\infty}\left|\lambda_{j}\right|^{p} \leq C\|f\|_{H \dot{K}_{q_{1}, \vec{b}}^{\alpha, p}}^{p}
\end{aligned}
$$

For $J$, let $x \in B_{k} \backslash B_{k-1}, b_{j}^{i}=\left|B_{j}\right|^{-1} \int_{B_{j}} b_{i}(x) d x, 1 \leq i \leq m, \vec{b}^{\prime}=\left(b_{j}^{1}, \ldots, b_{j}^{m}\right)$, we have

$$
\begin{aligned}
\mu_{\epsilon}^{\vec{b}}\left(a_{j}\right)(x)= & \left(\int_{0}^{\infty}\left|\int_{|x-y|<t} \prod_{i=1}^{m}\left(b_{i}(x)-b_{i}(y)\right) \frac{\Omega(x, x-y)}{|x-y|^{n-1-\epsilon}} a_{j}(y) d y\right|^{2} \frac{d t}{t^{3}}\right)^{1 / 2} \\
= & \left(\int_{0}^{|x|+2^{j}}\left|\int_{|x-y|<t} \prod_{i=1}^{m}\left(b_{i}(x)-b_{i}(y)\right) \frac{\Omega(x, x-y)}{|x-y|^{n-1-\epsilon}} a_{j}(y) d y\right|^{2} \frac{d t}{t^{3}}\right)^{1 / 2} \\
& +\left(\int_{|x|+2^{j}}^{\infty}\left|\int_{|x-y|<t} \prod_{i=1}^{m}\left(b_{i}(x)-b_{i}(y)\right) \frac{\Omega(x, x-y)}{|x-y|^{n-1-\epsilon}} a_{j}(y) d y\right|^{2} \frac{d t}{t^{3}}\right)^{1 / 2} \\
= & G+H .
\end{aligned}
$$

For $G$, noting that $y \in B_{j}, x \in B\left(0,2^{k}\right) \backslash B\left(0,2^{k-1}\right), j \leq k-3$, we know $|x-y| \sim|x| \sim|x|+2^{j}$.

Then, similar to the proof of Theorem 1, we obtain

$$
\begin{aligned}
G & \leq C \int_{B_{j}}\left|\int_{|x-y|}^{|x|+2^{j}} \frac{d t}{t^{3}}\right|^{1 / 2} \prod_{i=1}^{m}\left|b_{i}(x)-b_{i}(y)\right| \frac{|\Omega(x, x-y)|\left|a_{j}(y)\right|}{|x-y|^{n-1-\epsilon}} d y \\
& \leq \int_{B_{j}}\left|\frac{1}{|x-y|^{2}}-\frac{1}{\left(|x|+2^{j}\right)^{2}}\right|^{1 / 2} \prod_{i=1}^{m}\left|b_{i}(x)-b_{i}(y)\right| \frac{|\Omega(x, x-y)|\left|a_{j}(y)\right|}{|x-y|^{n-1-\epsilon}} d y \\
& \leq C 2^{j(1 / 2+\epsilon)} \int_{B_{j}} \frac{1}{|x|^{n+1 / 2}} \prod_{i=1}^{m}\left|b_{i}(x)-b_{i}(y)\right||\Omega(x, x-y)|\left|a_{j}(y)\right| d y \\
& \leq C \frac{2^{j(1 / 2+\epsilon)}}{|x|^{n+1 / 2}} \sum_{i=0}^{m} \sum_{\sigma \in C_{i}^{m}}\left|\left(\vec{b}(x)-\vec{b}^{\prime}\right)_{\sigma}\right| \int_{B_{j}}|\Omega(x, x-y)|\left|a_{j}(y)\right|\left|(\vec{b}(y)-\vec{b})_{\sigma^{c}}\right| d y \\
& \leq C \frac{2^{j(1 / 2+\epsilon)}}{|x|^{n+1 / 2}} \sum_{j=1}^{m-1} \sum_{\sigma \in C_{j}^{m}}\left(\int_{B_{j}}\left(\left|(\vec{b}(y)-\vec{b})_{\sigma^{c}}\right|\left|a_{j}(y)\right|\right)^{t} d y\right)^{1 / t}
\end{aligned}
$$




$$
\begin{aligned}
& \times\left(\int_{B}(|\Omega(x, x-y)|)^{r} d y\right)^{1 / r}\left|\left(\vec{b}(x)-\vec{b}^{\prime}\right)_{\sigma}\right| \\
& \leq C \frac{2^{j\left(1 / 2+\epsilon+n\left(1 / t+1 / r-1 / q_{1}-\alpha\right)\right)}}{|x|^{n+1 / 2}} \sum_{i=0}^{m} \sum_{\sigma \in C_{i}^{m}}\left\|\vec{b}_{\sigma}\right\|_{B M O}\left|\left(\vec{b}(x)-\vec{b}^{\prime}\right)_{\sigma}\right|, \\
& H \leq\left(\int_{|x|+2^{j}}^{\infty}\left|\int_{|x-y|<t} \prod_{i=1}^{m}\left(b_{i}(x)-b_{i}(y)\right)\right| \frac{\Omega(x-y, x)}{|x-y|^{n-1-\epsilon}}-\frac{\Omega(x, x)}{|x|^{n-1-\epsilon}}\left|a_{j}(y) d y\right|^{2} \frac{d t}{t^{3}}\right)^{1 / 2} \\
& \leq \int_{B_{j}} \frac{1}{|x|+2^{j}}\left|\frac{\Omega(x-y, x)}{|x-y|^{n-1-\epsilon}}-\frac{\Omega(x, x)}{|x|^{n-1-\epsilon}}\right| \prod_{i=1}^{m}\left|b_{i}(x)-b_{i}(y)\right|\left|a_{j}(y)\right| d y \\
& \leq C \frac{1}{|x|+2^{j}} \sum_{i=0}^{m} \sum_{\sigma \in C_{i}^{m}}\left|\left(\vec{b}(x)-\vec{b}^{\prime}\right)_{\sigma}\right| \int_{B_{j}}\left|\frac{\Omega(x-y, x)}{|x-y|^{n-1-\epsilon}}-\frac{\Omega(x, x)}{|x|^{n-1-\epsilon}}\right|\left|a_{j}(y)\right|\left|(\vec{b}(y)-\vec{b})_{\sigma^{c}}\right| d y \\
& \leq C \frac{1}{|x|+2^{j}} \sum_{i=0}^{m} \sum_{\sigma \in C_{i}^{m}}\left|\left(\vec{b}(x)-\vec{b}^{\prime}\right)_{\sigma}\right|\left(\int_{B_{j}}\left|\frac{\Omega(x-y, x)}{|x-y|^{n-1-\epsilon}}-\frac{\Omega(x, x)}{|x|^{n-1-\epsilon}}\right|^{r} d y\right)^{1 / r} \\
& \times\left(\int_{B_{j}}\left(\left|a_{j}(y)\right| \mid(\vec{b}(y)-\vec{b})_{\sigma^{c}}\right)^{t} \mid d y\right)^{1 / t} \\
& \leq C \frac{1}{|x|+2^{j}} \sum_{i=0}^{m} \sum_{\sigma \in C_{i}^{m}}\|a\|_{L^{q_{1}}}\left|B_{j}\right|^{1 / t-1 / q_{1}}\left\|\vec{b}_{\sigma^{c}}\right\|_{B M O}\left|\left(\vec{b}(x)-\vec{b}^{\prime}\right)_{\sigma}\right|\left(\frac{1}{2^{j}}+\int_{B_{j}} \frac{\omega_{r}(\delta)}{\delta} d \delta\right) \\
& \leq C \frac{2^{j n\left(-1 / q_{1}+1 / t-\alpha\right)}}{|x|+2^{j}} \sum_{i=0}^{m} \sum_{\sigma \in C_{i}^{m}}\left\|\vec{b}_{\sigma}\right\|_{B M O}\left|\left(\vec{b}(x)-\vec{b}^{\prime}\right)_{\sigma}\right|,
\end{aligned}
$$

thus

$$
\begin{aligned}
& \left\|\mu_{\epsilon}^{\vec{b}}\left(a_{j}\right) \chi_{k}\right\|_{L^{q_{2}}} \\
& \leq C 2^{j\left(1 / 2+\epsilon+n\left(1-1 / q_{1}-\alpha\right)\right)} \sum_{i=0}^{m} \sum_{\sigma \in C_{i}^{m}}\left\|\vec{b}_{\sigma c}\right\|_{B M O}\left[\int_{C_{k}}\left(|x|^{-(n+1 / 2)}\left|\left(\vec{b}(x)-\vec{b}^{\prime}\right)_{\sigma}\right|\right)^{q_{2}}\right]^{1 / q_{2}} \\
& \quad+C 2^{j n\left(1-1 / q_{1}-\alpha\right)} \sum_{i=0}^{m} \sum_{\sigma \in C_{i}^{m}}\left\|\vec{b}_{\sigma^{c}}\right\|_{B M O}\left[\int_{C_{k}}\left(|x|^{-1}\left|\left(\vec{b}(x)-\vec{b}^{\prime}\right)_{\sigma}\right|\right)^{q_{2}}\right]^{1 / q_{2}} \\
& \leq C 2^{j\left(1 / 2+\epsilon+n\left(1-1 / q_{1}\right)-\alpha\right)+k n\left(1 / q_{1}-\epsilon / n-1\right)}\|\vec{b}\|_{B M O} \\
& \quad+C 2^{j n\left(1-1 / q_{1}-\alpha\right)+k n\left(1 / q_{1}-\epsilon / n-1 / n\right)}\|\vec{b}\|_{B M O} .
\end{aligned}
$$

For the sake of simplicity, we denote

$$
\begin{aligned}
& W(j, k) \\
& =2^{j\left(1 / 2+\epsilon+n\left(1-1 / q_{1}\right)-\alpha\right)+k n\left(1 / q_{1}-\epsilon / n-1\right)} \\
& \quad+2^{j n\left(1-1 / q_{1}-\alpha\right)+k n\left(1 / q_{1}-\epsilon / n-1 / n\right)},
\end{aligned}
$$

then

$$
\left\|\mu_{\epsilon}^{\vec{b}}\left(a_{j}\right) \chi_{k}\right\|_{L^{q_{2}}} \leq C\|\vec{b}\|_{B M O} W(j, k)
$$


we obtain

$$
\begin{aligned}
J & \leq C\|\vec{b}\|_{B M O}^{p} \sum_{k=-\infty}^{\infty} 2^{k \alpha p}\left(\sum_{j=-\infty}^{k-3}\left|\lambda_{j}\right| W(j, k)\right)^{p} \\
& \leq \begin{cases}C\|\vec{b}\|_{B M O}^{p} \sum_{j=-\infty}^{\infty}\left|\lambda_{j}\right|^{p} \sum_{k=j+3}^{\infty} W(j, k)^{p}, & 0<p \leq 1, \\
C\|\vec{b}\|_{B M O}^{p} \sum_{j=-\infty}^{\infty}\left|\lambda_{j}\right|^{p}\left[\sum_{k=j+3}^{\infty} W(j, k)^{p / 2}\right]\left[\sum_{k=j+3}^{\infty} W(j, k)^{p^{\prime} / 2}\right]^{p / p^{\prime}}, & p>1\end{cases} \\
& \leq C\|\vec{b}\|_{B M O}^{p} \sum_{j=-\infty}^{\infty}\left|\lambda_{j}\right|^{p} \leq C\|\vec{b}\|_{B M O}^{p}\|f\|_{H \dot{K}_{q_{1}, \vec{b}}^{\alpha, p}}^{p}
\end{aligned}
$$

This completes the proof of Theorem 2 .

Remark Theorem 2 also holds for nonhomogeneous Herz-type spaces.

\section{Competing interests}

The authors declare that they have no competing interests.

\section{Authors' contributions}

In this paper, WY carried out the $\left(H_{\vec{b}}^{p}, L^{p}\right)$ and $\left(H \dot{K}_{q, \vec{b}}^{\alpha, p}, \dot{K}_{q}^{\alpha, p}\right)$-type boundedness for the multilinear commutator related to the Marcinkiewicz operator with variable kernels. YH, QL, JZ, XW participated in the analysis. All authors read and revised the final manuscript.

\section{Author details}

${ }^{1}$ College of Electrical and Information Engineering, Hunan University, Changsha, Hunan 410082, P.R. China. ${ }^{2}$ School of Electrical and Automation Engineering, Hefei University of Technology, Hefei, Anhui 230009, P.R. China.

\section{Acknowledgements}

This work was supported by the National Natural Science Funds of China for Distinguished Young Scholar under Grant No. 50925727, National Natural Science Foundation of China under Grant No. 60876022, The National Defense Advanced Research Project Grant No. C1120110004, Hunan Provincial Science and Technology Foundation of China under Grant No. $2010 J 4$ and 2011 JK2023, the Key Grant Project of Chinese Ministry of Education under Grant No. 313018.

Received: 30 March 2012 Accepted: 3 December 2012 Published: 27 December 2012

\section{References}

1. Chanillo, S: A note on commutators. Indiana Univ. Math. J. 31, 7-16 (1982)

2. Coifman, R, Rocherg, R, Weiss, G: Factorization theorem for Hardy space in several variables. Ann. Math. 103, 611-635 (1976)

3. Alvarez, J: Continuity properties for linear commutators of Calderón-Zygmund operators. Collect. Math. 49, 17-31 (1998)

4. Garcia-Cuerva, J, Herrero, ML: A theory of Hardy spaces associated to Herz spaces. Proc. Lond. Math. Soc. 69, 605-628 (1994)

5. Lu, S, Yang, D: The local versions of $H^{P}\left(R^{n}\right)$ spaces at the origin. Stud. Math. 116, 147-158 (1995)

6. Lu, SZ, Yang, DC: The decomposition of the weighted Herz spaces and its applications. Sci. China Ser. A 38, 147-158 (1995)

7. Lu, SZ, Yang, DC: The weighted Herz type Hardy spaces and its applications. Sci. China Ser. A 38, 662-673 (1995)

8. Liu, LZ: The continuity of commutators on Triebel-Lizorkin spaces. Integral Equ. Oper. Theory 49(1), 65-76 (2004)

9. Liu, LZ, Wu, BS: Weighted boundedness for commutator of Marcinkiewicz integral on some Hardy spaces. Southeast Asian Bull. Math. 28, 643-650 (2004)

10. Pérez, C: Endpoint estimate for commutators of singular integral operators. J. Funct. Anal. 128, 163-185 (1995)

11. Pérez, C, Trujillo-Gonzalez, R: Sharp weighted estimates for multilinear commutators. J. Lond. Math. Soc. 65, 672-692 (2002)

12. Stein, EM: Harmonic Analysis: Real Variable Methods, Orthogonality and Oscillatory Integrals. Princeton University Press, Princeton (1993)

13. Torchinsky, A, Wang, S: A note on the Marcinkiewicz integral. Colloq. Math. 60/61, 235-243 (1990)

14. Yu, WX, Liu, LZ: Sharp weighted inequalities for multilinear commutator of Marcinkiewicz operator (to appear)

15. Yu, X, Tao, XX: The boundedness of Marcinkiewicz integration with variable kernels on Herz-type Hardy space. J. Ningbo Univ. (NSEE) 19, 200-203 (2006) 